\title{
AVC hémorragique : diagnostic guidé par les résultats du champ visuel
}

\author{
Nicole Riese, OD, FAAO \\ Optométriste traitante, \\ VA New Jersey Health \\ Care System \\ Melissa Bailey, MD \\ Faculté de médecine, \\ University of Miami \\ Ce document est le fruit d'un \\ travail soutenu par des ressources \\ et l'utilisation des installations \\ du New Jersey Health Care \\ System, du Michael E. DeBakey \\ Veterans Administration Medical \\ Center et de la Katy Veterans \\ Administration Outpatient Clinic. \\ Le contenu ne représente pas le \\ point de vue du département des \\ Anciens Combattants des États- \\ Unis ni du gouvernement des \\ États-Unis.
}

\begin{abstract}
Résumé
Ce rapport de cas porte sur un patient qui a subi un accident vasculaire cérébral hémorragique et qui s'est présenté au service de consultations ophtalmologiques avec une perte de champ visuel, que le patient a interprétée comme un trouble de la vue et un mal de tête grave. La perte de champ visuel a consisté en une hémianopsie incomplète homonyme accompagnée d'une défectuosité supérieure absolue qui correspondait bien à l'hémorragie du lobe temporal droit détectée par une tomodensitométrie de la tête. Ce cas expose l'importance pour tous les fournisseurs de soins oculovisuels de reconnaître les symptômes de l'AVC et de comprendre les délais et les protocoles de référence, ainsi que la valeur prédictive élevée des champs visuels.
\end{abstract}

\section{MOTS CLÉS :}

AVC hémorragique, hémianopsie homonyme, mal de tête, malformation artérioveineuse

\section{INTRODUCTION}

Les accidents vasculaires cérébraux sont, tout juste après les cardiopathies ischémiques, la cause la plus courante de décès dans le monde; en 2010, on estime que 5,9 millions de personnes sont mortes des suites d'un accident vasculaire cérébral ${ }^{1}$. Un accident vasculaire cérébral est défini comme un infarcissement ou une hémorragie dans le cerveau, la rétine ou la moelle épinière causant une anomalie neurologique focale soudaine qui dure plus de 24 heures ou qui, à l'imagerie, montre un infarcissement ou une hémorragie correspondant à des symptômes de n'importe quelle durée ${ }^{1}$. Les symptômes de l'accident vasculaire cérébral varient bien qu'ils comprennent habituellement une faiblesse unilatérale, des engourdissements, des changements visuels (dont la diplopie), l'altération de la parole, l'ataxie et des vertiges non attribuables à de l'hypertension orthostatique ${ }^{1}$. Une étude récente révèle que des changements visuels attribués à un accident vasculaire cérébral se manifestent chez $48 \%$ des patients admis pour un accident vasculaire cérébral et chez $60 \%$ des survivants d'un accident vasculaire cérébral; les changements visuels comprennent l'atteinte de la vision centrale (56\%), des mouvements oculaires anormaux (40\%), la perte de champ visuel (28\%), l'inattention visuelle (27\%) et les troubles de la perception visuelle $(5 \%)^{2}$.

Il peut s'avérer difficile de déceler un accident vasculaire cérébral aigu chez un patient, surtout si les symptômes sont atypiques et variables, si l'on n'a pas accès aux appareils d'imagerie ou si les résultats semblent normaux. La perte de champ visuel, en particulier, est un outil puissant qui fournit des informations utiles permettant de prédire l'existence d'une pathologie. Un infarcissement ou une hémorragie postérieure au chiasma optique entraînera une perte bilatérale du champ visuel par rapport à la ligne médiane verticale. Une lésion entraînant une hémianopsie homonyme complète ne peut être localisée que si elle est postérieure au chiasma du côté contralatéral de la perte du champ visuel. Les hémianopsies incomplètes peuvent 
fournir plus d'information; brièvement, les anomalies congruentes ont tendance à être plus en position postérieure sur les voies optiques ${ }^{3}$. Par ailleurs, les anomalies du champ visuel supérieur ont tendance à résulter de dommages au lobe temporal tandis que les anomalies du champ visuel inférieur résultent de lésions au lobe pariétal ${ }^{3}$.

\section{RAPPORT DE CAS}

Un homme de 52 ans s'est présenté au service de consultations ophtalmologiques en raison d'une vision légèrement floue dans les deux yeux et d'un mal de tête grave. Cinq jours avant la consultation, le patient regardait la télévision et a éprouvé un mal de tête soudain du côté gauche associé à une photophobie. Il a classé subjectivement la douleur à 10/10. Au réveil, le lendemain matin, il a remarqué qu'il éprouvait une vision floue dans les deux yeux. Il a éprouvé un malaise semblable dans les années 1990 qui a été diagnostiqué comme une migraine. Au cours de l'examen, le patient a obtenu 20/20 avec l'œil droit, 20/25 avec l'œil gauche, et l'on a soupçonné une anopsie du quadrant supérieur gauche dans la périmétrie de confrontation en recourant à l'échelle NIHSS (National Institutes of Health Stroke Scale) $)^{4}$. Le reste de l'examen du fond d'œil sous pupille dilatée a donné des résultats semblables à l'examen complet qu'il avait subi un mois plus tôt et qui n’avait rien révélé de pertinent. L'examen formel du champ visuel Humphrey Field Analyzer (HFA) en utilisant la stratégie 24-2 standard SITA (Carl Zeiss Meditec, Dublin, CA) a été réalisé. Il a permis de confirmer la présence d'une hémianopsie homonyme gauche incomplète, avec une anopsie absolue du quadrant gauche supérieur (figure 1). Une lésion dans le lobe temporal droit du patient a été soupçonnée et le patient a été envoyé au service des urgences avec une copie des résultats de l'examen.

Figure $1:(a, b)$ Examens du champ visuel effectués le jour de la consultation initiale. Une hémianopsie homonyme gauche incomplète et une anopsie absolue du quadrant gauche supérieur sont apparentes. L'étiologie neurologique était la cause la plus probable de ces anomalies du champ visuel.

a)

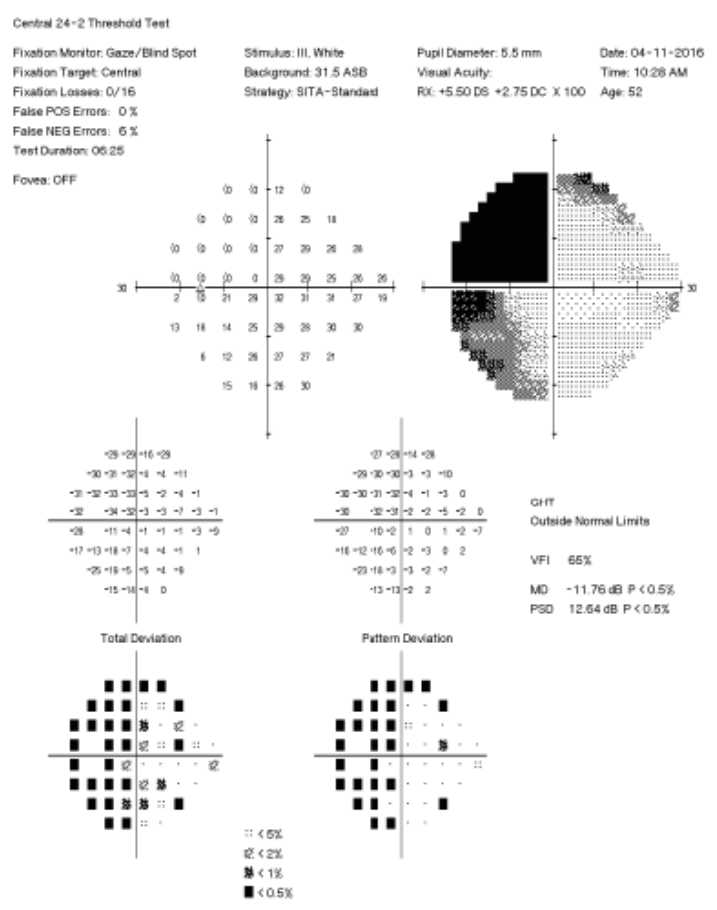

b)

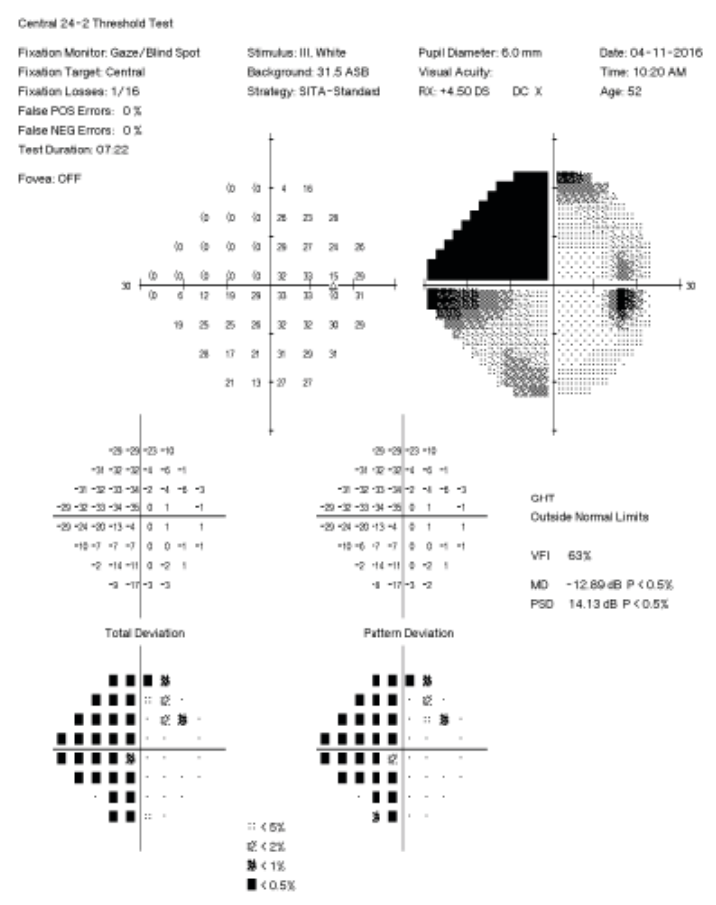

À l'urgence, une tomodensitométrie de la tête a révélé une importante hémorragie parenchymateuse dans le lobe temporal droit, un type particulier d'hémorragie intracrânienne, montrant une déviation de $4 \mathrm{~mm}$ vers la gauche de la ligne médiane (figures 2,3). La taille de la lésion a été mesurée à 6,1 x 3,3 x 1,8 cm. Le patient a été admis à l'unité des soins intensifs et surveillé de près. Une angiographie par tomodensitométrie et une imagerie par résonance magnétique avec et sans contraste ont été réalisées mais n'ont pas permis de repérer la source de l'hémorragie. 
L'examen complet en interne n'a pas non plus révélé l'étiologie de l'hémorragie intracrânienne, bien qu'une malformation artérioveineuse ait été l'étiologie présumée compte tenu du jeune âge du patient, de l'apparence et de l'emplacement de l'hémorragie et de l'absence d'autres problèmes médicaux. Environ quatre mois après le congé du patient, des examens externes subséquents ont révélé la cause de l'hémorragie intracrânienne.

Figure 2 : Vue axiale de l'IRM à pondération en diffusion. La région de l'hyposignal dense ou sombre du côté gauche de l'image correspond au lobe temporal droit du patient et représente la zone de l'hémorragie.

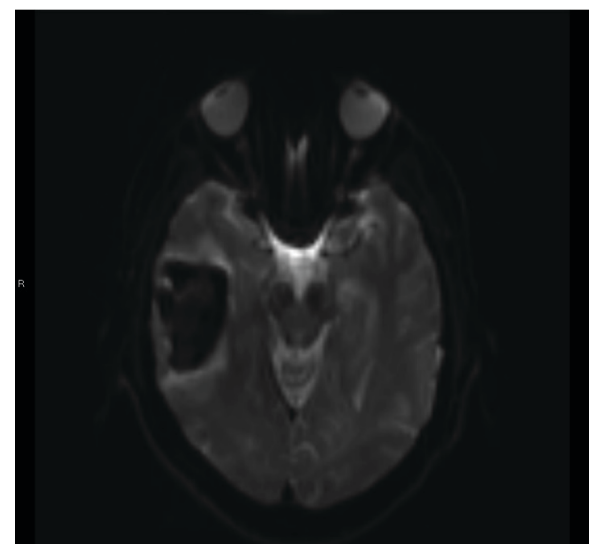

Figure 3 : Vue sagittale de l'IRM pondérée en T1. La zone des hypersignaux, ou zone lumineuse, indique l'emplacement et la taille de l'hémorragie dans le lobe temporal.

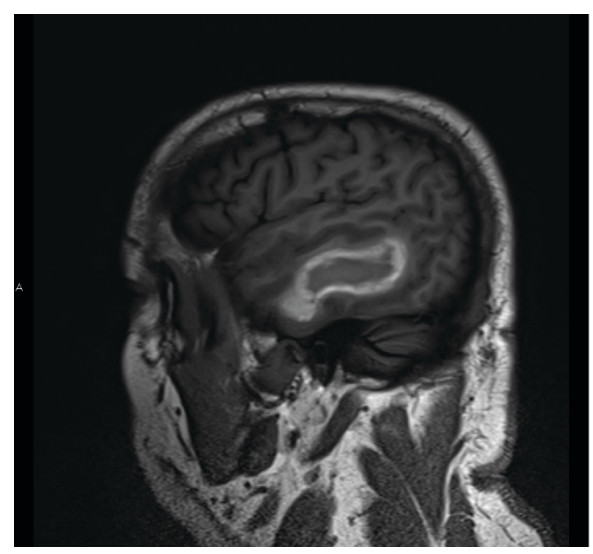

Environ un an après la consultation initiale, un angiogramme cérébral a révélé qu'une malformation artérioveineuse de grade 1 dans le lobe temporal droit selon l'échelle Martin-Spetzler était à l'origine de l'hémorragie intracrânienne, c'est-à-dire qu'une connexion anormale entre une artère et une veine contournait le lit capillaire et créait une zone sujette aux hémorragies chez le patient. Les malformations artérioveineuses de grade 1 sont de petite taille et présentent le risque chirurgical le plus faible; ce patient souhaitait que la correction chirurgicale de la malformation aide à prévenir l'occurrence d'une hémorragie future et une opération a été effectuée avec succès le mois suivant. Le patient continue d'être suivi au service de consultations pour basse vision et les examens répétés des champs visuels ont montré une anopsie absolue supérieure stable du quadrant gauche et une amélioration dans le quadrant inférieur. Comme l'anomalie du champ visuel a persisté pendant plus de 24 heures et qu'elle a été causée directement par l'hémorragie intracrânienne, il s'agit d'un accident vasculaire cérébral hémorragique. Le patient a cessé de conduire en raison de sa perte de champ visuel et a toujours de la difficulté à voir des objets sur son côté gauche, a plus de difficulté à lire et éprouve des problèmes d'équilibre. Il s'exerce toujours avec des prismes et est suivi par divers thérapeutes en réadaptation. 
DISCUSSION

Dans ce cas, la concordance des anomalies du champ visuel par rapport à la ligne médiane verticale suggérait fortement une étiologie neurologique. De plus, un examen oculaire complet a exclu tout trouble de la rétine ou du nerf optique qui aurait pu expliquer les résultats de l'examen du champ visuel. Par conséquent, les principaux diagnostics différentiels restants avant le renvoi du patient à l'urgence comprenaient une migraine accompagnée de manifestations ophtalmiques, une anomalie du champ visuel installée depuis longtemps et causés par un accident vasculaire cérébral ou un événement neurologique antérieur ou, encore, une nouvelle masse ou une autre lésion ou un infarcissement touchant probablement le lobe temporal droit. Même si le patient avait des antécédents de migraines, et il est possible qu'il ait eu une anomalie congruente ${ }^{3}$, cela se produit rarement de sorte que les étiologies sous-jacentes plus graves devaient être écartées. Il était peu probable que cette anomalie du champ visuel se soit installée depuis longtemps à la suite d'un accident vasculaire cérébral ou d'un événement neurologique antérieur étant donné que le patient a remarqué de nouveaux symptômes oculaires. Compte tenu de l'apparence des anomalies du champ visuel et des maux de tête connexes, le diagnostic différentiel le plus probable était un nouvel infarcissement ou une hémorragie affectant le lobe temporal droit, ce qui commande un renvoi urgent le jour même. Les maux de tête du patient particulièrement, compte tenu de leurs caractéristiques, laissaient supposer la présence d'une hémorragie cérébrale. Un mal de tête associé à une hémorragie intracrânienne se manifeste souvent de façon soudaine et peut être décrit comme un « mal de tête fulgurant », c'est-ç-dire un mal de tête grave qui atteint son intensité maximale en une minute ${ }^{5}$. Le mal de tête est généralement situé du même côté de la tête que l'hémorragie bien que, dans ce cas-ci, il se soit produit du côté opposé. Parmi les autres caractéristiques préoccupantes, mentionnons les nausées et les vomissements, l'altération du niveau de conscience, la raideur du cou, les crises d'épilepsie, l'hypertension sévère et les déficits neurologiques connexes comme la faiblesse ou l'affaissement unilatéral du visage; ce patient avait un déficit neurologique connexe constitué d'une perte du champ visuel'.

Un diagnostic d'hémorragie intracrânienne s'accompagne entre autres d'un taux de mortalité à un mois estimé à 35 à $50 \%$, d'un pronostic plus défavorable pour les hémorragies plus importantes, d'une baisse du niveau de conscience au moment de l'admission et d'une déviation de la ligne médiane ${ }^{7,8}$. Fait important, bien que l'hémorragie intracrânienne puisse être attribuée à diverses étiologies sous-jacentes, lorsque l'hémorragie intracrânienne est due à une rupture de la malformation artérioveineuse, l'issue est habituellement plus favorable ${ }^{9}$. Les patients qui ont une hémorragie intracrânienne associée à une malformation artérioveineuse ont tendance à être beaucoup plus jeunes et à présenter moins de facteurs de risque connus de maladie et d'accident vasculaire cérébral. Toutefois, même en tenant compte de ces variables, ces patients présentent un risque plus faible de décès et des taux plus élevés d'issue favorable à l'obtention de leur congé?.

Bien que la malformation artérioveineuse de ce patient n'ait pas été diagnostiquée après que l'hémorragie intracrânienne ne soit résorbée et que l'angiographie cérébrale ait été effectuée, d'autres patients peuvent avoir des problèmes préexistants connus ou présenter des anomalies que l'imagerie initiale peut révéler. Lorsqu'un patient est hospitalisé en raison d'une hémorragie intracrânienne causée par une malformation artérioveineuse, la pratique courante dans certains hôpitaux consiste à recommander une résection chirurgicale au cours de l'hospitalisation initiale $^{10}$. Les malformations artérioveineuses ont un taux de rupture annuel estimé entre 1 et $4 \%$. Les résultats de l'étude ARUBA (étude randomisée sur les malformations artérioveineuses non rompues du cerveau) suggèrent que les malformations artérioveineuses non rompues devraient être abordées avec précaution en adoptant une prise en charge médicale plutôt que chirurgicale ${ }^{10}$. Néanmoins, dans ce cas, en raison de la rupture antérieure de la malformation artérioveineuse, le patient a choisi de subir une résection chirurgicale.

Comme on l'a démontré dans ce cas, la qualité de vie est souvent affectée après une perte du champ visuel homonyme. Dans certaines provinces, les patients atteints d'hémianopsie homonyme ne peuvent plus conduire en raison des exigences propres au champ visuel et, dans les régions qui n'ont pas d'exigences à ce propos, il a été démontré que ces patients ont une capacité réduite à détecter les piétons et à réagir à des événements inattendus ${ }^{3}$. De nombreux facteurs liés à la perte de champ visuel contribuent à réduire la vitesse de lecture et la compréhension chez de nombreux patients ${ }^{3}$. De nombreux patients atteints d'hémianopsie homonyme peuvent être dirigés vers d'autres services, notamment de basse vision pour la thérapie avec prisme, de réadaptation pour conduire un véhicule ou d'ergothérapie ${ }^{3}$. Il faut aussi informer le patient que, dans la plupart des cas d'hémianopsie homonyme, les anomalies du champ visuel peuvent s'estomper au cours d'un mois ou deux, mais qu'il est peu probable qu'elles s'estompent après six mois, à moins qu'une cause sous-jacente soit corrigée ${ }^{3}$.

Sans les résultats de l'examen du champ visuel chez ce patient, il est probable que l'hémorragie intracrânienne n'aurait pas été diagnostiquée en temps opportun. Ce cas met en évidence l'importance d'effectuer en toute urgence 
un examen du champ visuel en présence de maux de tête atypiques ou nouveaux et particulièrement lorsque le patient peut consulter un optométriste avant de consulter un médecin de premier recours. Dans ce cas précis, la perte de champ visuel était suffisamment importante pour être détectée avant qu'un examen automatisé ne soit effectué bien que, dans d'autres cas, un examen automatisé puisse être le seul moyen de détecter la perte de champ visuel. Lorsqu'un patient présente des symptômes et qu'une perte du champ visuel indiquant une étiologie neurologique est découverte, il faut le diriger vers le service des urgences pour qu'il subisse des tests et pour qu'on le prenne en charge immédiatement car sa vie pourrait être en danger.

\section{RÉFÉRENCES}

1. Hankey GJ. Stroke. Lancet. 2017; 389: 641-654. DOI: 10.1016/S01406736(16)30962-X.

2. Rowe FJ, Hepworth LR, Howard C, Hanna KL, Cheyne CP, Currie J. High incidence and prevalence of visual problems after acute stroke: An epidemiology study with implications for service delivery. PLOS ONE 2019; 14(3): 1-16. DOI: 10.1371/journal.pone.0213035

3. Goodwin D. Homonymous hemianopia: challenges and solutions. Clin Ophthalmol. 2014; 8: 1919-1927. DOI: 10.2147/OPTH.S59452

4. National Institute of Neurological Disorders and Stroke. NIH Stroke Scale. https://www.ninds.nih.gov/sites/default/files/NIH Stroke_Scale_Booklet.pdf. Accessed August 26, 2019.

5. Ducros A, Bousser MG. Thunderclap headache. BMJ. 2013; 346e: 8557. DOI: 10.1136/bmj.e8557

6. An SJ, Kim TJ, Yoon BW. Epidemiology, risk factors, and clinical features of intracerebral hemorrhage: an update. J Stroke. 2017; 19(1): 3-10. DOI: 10.5853/jos.2016.00864
7. Suthar NN, Patel KL, Saparia C, Parikh AP. Study of clinical and radiological profile and outcome in patients of intracranial hemorrhage. Ann Afr Med. 2016; 15: 69-77. DOI: 10.4103/1596-3519.176259

8. Morotti A, Goldstein JN. Diagnosis and management of acute intracerebral hemorrhage. Emerg Med Clin North Am. 2016; 34(4): 883-899. DOI: 10.1016/j.emc.2016.06.010

9. Murthy SB, Omran SS, Gialdini G, et al. Outcomes after intracerebral hemorrhage from arteriovenous malformations. Neurology. 2017; 88: 1882-1888. DOI: 10.1212/WNL.0000000000003935

10. Mohr JP, Parides MK, Stapf C, et al. Medical management with or without interventional therapy for unruptured brain arteriovenous malformations (ARUBA): a multicenter, non-blinded, randomised trial. Lancet. 2014;383(9917):614-621. DOI: 10.1016/S01406736(13)62302-8 